\title{
Using of Advanced Communication Technology in the Construction Projects During the Implementation Stage
}

\author{
Wadhah A. Hatem ${ }^{1}$, Hafadh I. Naji ${ }^{2}$, Zainab A. Abd Alkreem ${ }^{3}$ \\ ${ }^{1}$ Assistant Professor, Baquba Technical Institute, Middle technical University, \\ ${ }^{2}$ Assistant Professor, ${ }^{3}$ M.Sc.Student, Department of Civil Engineering, Engineering College, \\ University of Diyala \\ 1'wadhah1970wadhah@gmail.com, ${ }^{2}$ hafizibrahem1973@ gmail.com, ${ }^{3}$ zainabeng63@yahoo.com
}

\begin{abstract}
Iraq is a wide country in the area and construction projects have been distributed in many places, therefore this research studies the ability to use advanced communication tools such as computer-mediated communication instead of traditional communication (Face To Face). Revit application which based on BIM technology have been used, this application helped in exchange design information between designer and site engineer for in making decisions, solving problems, reduce wasted time and reduce the expenses that occur as a result of the use of FTF communication .The results concluded from this research are that communication quantity in FTF was more than in CMC where Total Number of Words (TNOW) were in FTF 303 while in CMC 246, The percentage of Number of Work Related Words said in CMC is higher than in FTF, it was $87 \%$ in CMC and $81 \%$ in FTF of the (TNOW), it is indicating that communication in CMC more productive than communication in FTF, in addition to that the working time in CMC were (262 seconds every 5-minutes) slightly higher than in FTF were (252 seconds every 5-minutes), this explains that $\mathrm{CMC}$ was slightly more productive than FTF . Wasted time for FTF consistently higher than wasted time in CMC , where the averages of wasted time spent for every 5 minutes were 32 second in FTF and 21 in CMC , this is due to that FTF was easier than CMC in terms of social speech. To identify degree collaboration between users in two methods by the total number of exchanges in CMC was higher than in FTF because most of the persons were having more interaction when using CMC.
\end{abstract}

Keywords: Communication , Collaboration, FTF ,CMC.

Paper History: Received: (6/4/2016), Accepted: (12/12/2016)

\section{Introduction}

The building and construction of industry play a substantial role in evolving and accomplishing the objectives of society [1].Construction industry generally and especially in Iraq has been characterized by uncertainty, and adversarial attitudes for a long time because of the wide variety of disciplines, expertise, professional skills, educational background, laptop acquaintance, and workplace among the project members. All these impede the information administration and communication of the project team. Also, the production environment (construction work site) is often remotely managed by designers' office which has a big effect on the accomplishment of complete design and construction, this would lead to the additional effort of information management and communication process between building contractors and design teams, and also leads to difficult access to project information by project participants in construction sites[2]. For eliminating this obstacle, it is necessary developing the collaboration and interaction between members. The main ingredient in this reaction is the communication operation and information administration which have been determined as a factor for the efficiency participant collaboration.

This research contributes to a best understanding of the issues related to information flow management when using centralized platforms. It will permit us to demonstrate the degree of usefulness and practicality of BIM (Building Information Modeling) as technologies and processes which are considered the best solution for poor information management processes within the construction project network. The research adopted resolving problem task which is related to find best solution when designers collaborate with site engineers in two forms of communication models face to face (FTF) and computer mediated communication (CMC) [3]. Autodesk Revit Architecture version 2015 has been used in this research which has many 
facilitates to make people who are geographically distributed in different areas and sites collaborate.

\section{Related Works}

This section explains role of effective communication in project success and communication methods ( FTF and CMC).

\subsection{Role Of Effective Communication In Project Success}

Effective communication is essential to project management .It makes a bond among various participants involved in a venture, sharing different regulatory and cultural backgrounds , various levels of experiment, perspectives and interests in the venture implementation [4]. The studies explained that 55 as percent of venture managers have agreed that active communications to all participants is the most effective element in venture management [5], venture managers \& venture team individuals spend about 90 as percent of their working time involved in some practice of communication, like reading emails, writing reports, meetings, or talking to project participants [6].They determined that almost (75-90) \% of venture manager's time spent in the building industry in communication $[7,8]$.

PMI's 2013 Pulse of the Profession TM report shows that for each one billion US\$ expended on a venture (135 million) is at hazard. More research on significance of active communications displays that a startling 56 as percent is at risk due to ineffective communications [5]. Not only is an organization risking dollars, but it is risking the project success rates. Studies proved that ineffective communications lead to less successful projects; in organizations that are less effective communication, effective communicators report significantly fewer projects that meet original goals, come on time, and complete within budget [5] (See Figure 1).

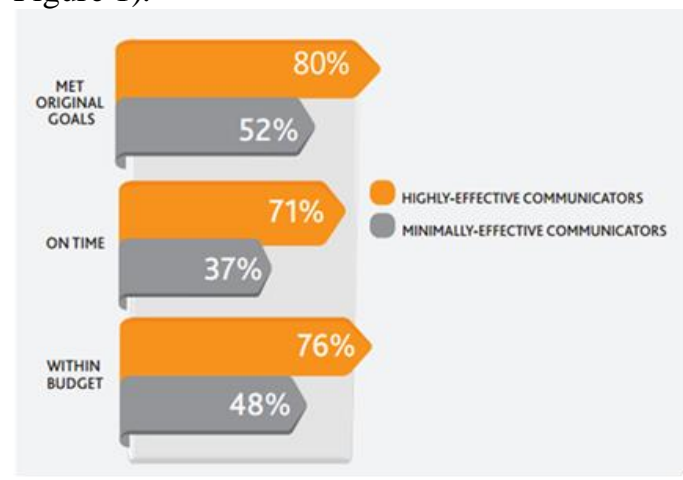

Figure 1: Organizations that communicate more effectively have more successful projects [5].

\subsection{Communication Methods (FTFand CMC )}

There are two kinds of communication methods, traditional method which defined Face to Face (FTF), it is a dialogue that one has while being FTF with the other person .During this kind of communication, a person can hear and see the non-oral communication transfer by the transmitter and respond with feedback immediately [9] and modern method which defined Computer -Mediate Communication and in other hands defined Virtual team, Virtual teams can be defined as: "Teams whose individuals are operating across locations and temporal boundaries using technology in varying degrees to accomplish an interdependent task" [10] . According to Leenders et al. "virtual teams are set of persons collaborating in the implementation of a specific project, they are distributed geographically and mostly temporally [11] .

(CMC) teams are significantly different from traditional teams, from these differences:-

- (CMC) teams are physically detached and relied on technologies using between them while traditional teams (face to face interactions) work under the same roof in close physical proximity [12].

- (CMC) teams were more complex of Face to face teams in terms of geographical distribution and communication media ; because

- they work across boundaries different in time, space and organization and

- they use technology in communication $[13,14]$.

- In the (CMC) teams, tasks must be highly structured, while the traditional teams could be structured simply by individuals of the team with each other [15].

- (Communication in (CMC) teams is more restricted than face to face (FTF) teams because different of cultures ,diversity, language and the nature of tasks [16] .

- Andres predicted that interaction quality in a face to face teams is greater than (CMC) teams where increase using the verbal and non-verbal signal to enhance direct feedback in FTF teams [17]

- In an innovation network; individuals' work in "virtual organizations" is unlimited by place and time, and communication can be facilitated by IT, while the innovation process 
in "traditional organization", is more limited by location and time where it happens within the scope of working hours and physical offices [18].

- They found that communication using rich media achieved higher task performance than communication using less rich media [19].

- In terms of conflict, they found that conflict diverse in virtual teams more of FTF teams due to its diverse and dispersed nature [20].

- They compared 11 traditional face to face teams with 13 teams using an asynchronous technology, they found that face to face teams are more cohesive and satisfied with the team's decision and outcome process than VT teams [21].

\section{Experimental Work}

- Experiments were performed on building model Cultural Center Appendex at the College of Engineering as show in Figure 2.

-Sixteen Experiments implemented are used by two methods: Face-to-Face meetings and computer mediated communication (CMC) meetings

- Experiments were performed by a team of two users, users which work in FTF are the same in $\mathrm{CMC}$, on the same modeling but tasks are different in FTF and CMC.

-Teams discuss the task which includes solving design problems discovered by the site engineering before implementation from an architectural perspective, for example, exterior walls, interior walls, the roof, the floor, doors and windows, etc..

- In some experiments, teams are using a wide area network (WAN) to connect and in other experiments they are using local area network (LAN) and they use the programs Revit and Skype for communication.

- The use of cameras to record the work and the use of a program Camtasia to record the screen and note the changes that made on the model.

- Data were obtained by referring to the video recordings, as well as screen recordings.

- Analyzing the results for both FTF and CMC to extract the most important parameters, such as the number of words, work related word, working time, wasting time, the degree of collaboration and team productivity.

The approach of experiments which is structured from teams of two people, each team implemented two tasks. Task 1 is carried out by FTF team and consisted of 2 worksets (i.e. sectors): the exterior walls and doors \& windows . Task 2 is carried out by CMC team and also consisted of 2 worksets: interior wall and ceiling \& floor. It is necessary that FTF tasks should be different from CMC tasks. As previously mentioned that the team which was implemented experiment in FTF is the same in $\mathrm{CMC}$ in all experiments, so if the tasks in FTF and CMC were the same, the answer will be known to each user, Thus the discussion would be useless, so the tasks were designed to be in the same difficult level in both methods. To implement tasks, both users accessed to model in Revit via the same PC In FTF, while in CMC each user has special model on his computer..

Workset can be established by multiple users. In this model, workset was established by two users "admin" and "Zainb" as shown in Figure 3. Workset which consists of floors ,stairs and exterior wall, all these are owned by a user called "admin " while workset which consists of col, door \& windows and interior wall, the owner of these is a user called "Zainb" this shows collaborative work in CMC experiments . In this form of collaborative work, we need to a permission request, for example, If the user "Zainb" wants to change in the exterior walls, she will be required a license request from the user" admin" because the exterior walls are owned by user "admin" as show in Figure 4. But in most of the experiments done, worksets were owned by designer, in other word, only designer can change on the model.

For both FTF and CMC tasks, the users have to work using the 3D computer model of the building, some tasks were real or actually occurred during the execution, but some tasks were virtual and have been proposed by some engineers. The time required to complete each task was 30 minutes, it is difficult doing experiment in time less than 30 minutes because there are some design changes, also it is difficult doing experiment in time more than 30 minutes because there is a difficulty taking more than an hour from volunteers in both tasks.

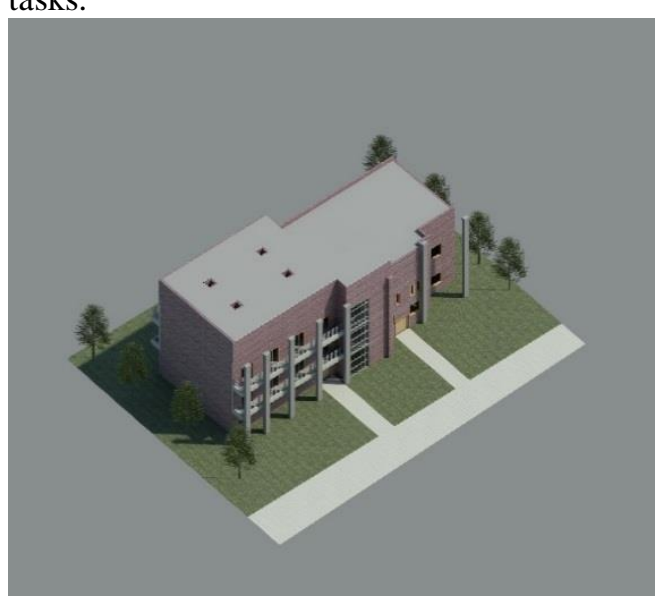

Figure 2: Culture Center Appendex 


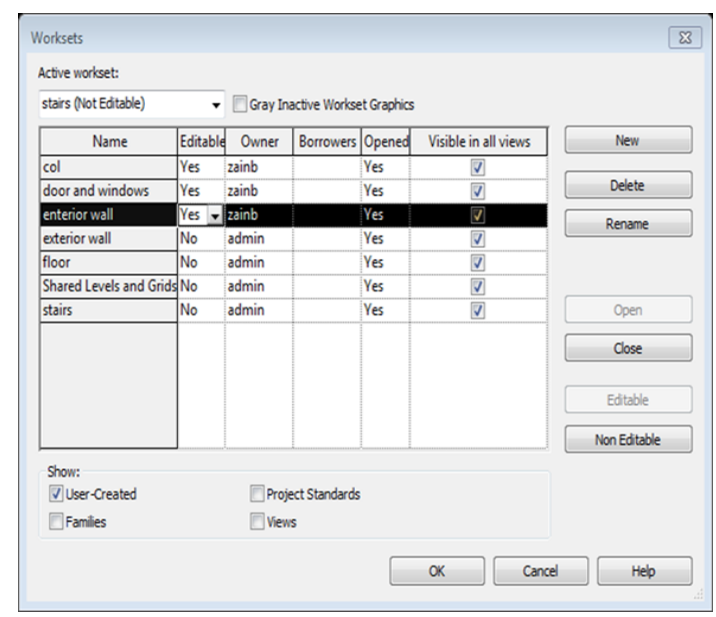

Figure 3: window of workset in Revit

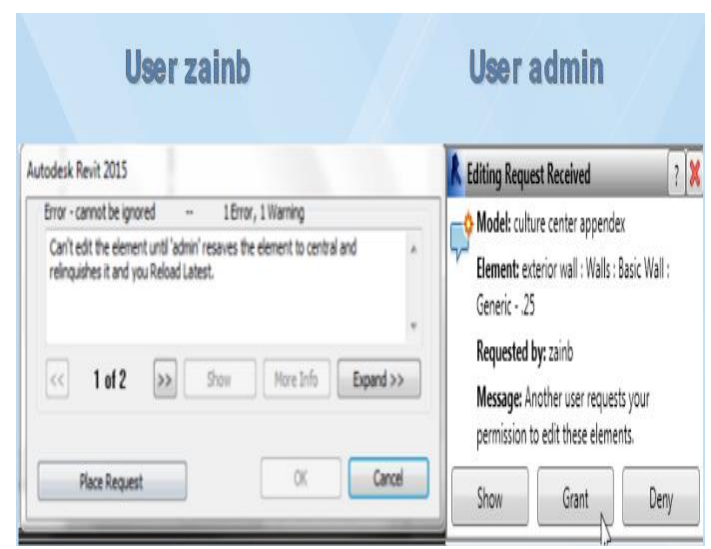

Figure 4: show request permission between user

\section{Results and Discussion}

\subsection{Total Number of Words (TNOWs)}

The amount of the (TNOWs) which has been spoken between the users during the experiments is a measure of communication done [22], but it does not give an indication of the team productivity because some words are not related to the work ,in addition to that, the total number of words refers only to the quantity of communication not the quality, the dependent's result are averages of the 16 experiments. Figure 5, shows the total number of words in FTF and CMC communication. The average of (TNOWs)in FTF has recorded 303 words per 5-minutes while in CMC the total number of words has recorded 246 words, the (TNOWs)said by each team in FTF is $23 \%$ higher than in CMC during the six 5-minute intervals, this is stated by the statistical analysis (the Student's t-distribution), where there are the significant statistical difference between mediums of the two groups. It is obvious that in FTF the participants spoke words more than in CMC, and when analyzing the transcripts, it was clear that the users were more concentrating on their task in CMC than FTF.
The value of $t$ ("t Stat") is 5.82 , this value is compared to the value of "t Critical two-tail" (tcrit) of 2.13145 based on $\alpha=0.05$ (onesided), and 15 df . Because("t Stat") > tcrit $(5.82>2.13)$, the null hypothesis is rejected at the $95 \%$ level of confidence. Thus, the words medium in FTF is measured greater than the words medium in CMC at the $95 \%$ level of confidence.

Figure 5 shows average number of words said in all the experiments, divided into five minute intervals. Both curves have the same trend in most of the time intervals. It can be watched that in FTF the users discuss most of the items at the beginning of the experiment, after agreement on items, the designer would start to change designs on the computer, It is obvious the total number of words was at a high level in the start of the experiment but this average was decreasing gradually, till the last five minutes, the curve has noted an increase slowly. In the CMC each work set was discussed and then the designer started to change on the model, so that a high level of the total number of words were recorded during the first 5 minutes, and then started to go down for 15 minutes then raised again in the minute 20 where users started discussing the second work set. After that it went down again.

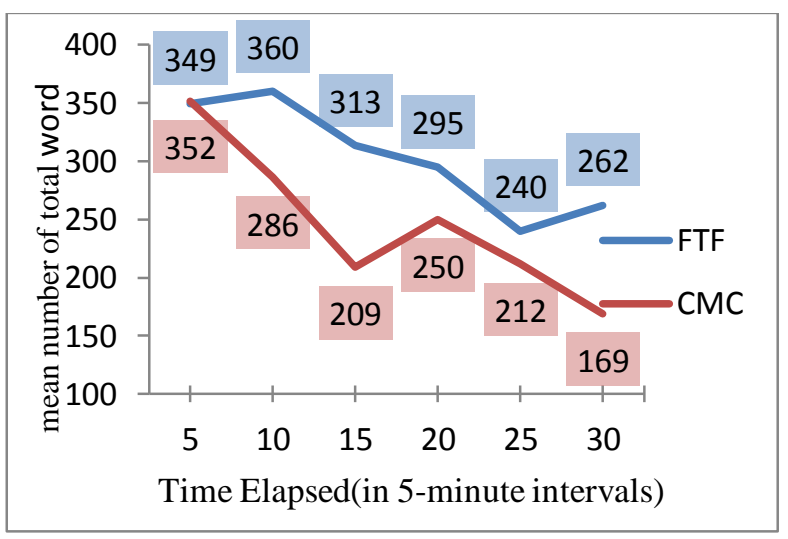

Figure 5: Total Number Of Words in FTF and CMC

\subsection{Total Number of Work Related Words (TNWRWs)}

In the previous paragraph, the researcher discussed the (TNOW) in each method of communication the FTF and CMC. It is obvious that (TNOW) in FTF was more than in CMC insignificant statistical difference , The reason of this is the need of more social communication when persons are gathered. This can be detected by analyzing the texts to determine the (TNWRWs). Like before, the results are the averages of 16 experiments 
showing as words spoken during 5 minute periods. Over the six 5-minute intervals, FTF recorded an average of 245 words for every 5minute while CMC recorded 214. The percentage of (TNWRWs) said in CMC is higher than in FTF, it was $87 \%$ in CMC and $81 \%$ in FTF of the (TNOW) both CMC and FTF , this is stated by the statistical analysis (the Student's t-distribution), where there are the significant statistical difference between mediums of the two groups.

The calculation value of $t$ ("t Stat") is 2.91,this value is compared to the value of "t Critical two-tail" (tcrit) of 2.13 based on $\alpha=0.05$ (one-sided), and $15 \mathrm{df}$. Because ("t Stat") was bigger than tcrit (2.91> 2.13). Thus, (TNWRWs) medium of FTF is measured higher than the (TNWRWs) medium of CMC at the $95 \%$ level of confidence. As shown below in Figure 6, the style of the curves of (TNWRWs) is very similar to the curve of the total number of wordsExplanations of the big (TNOW) spoken in FTF, the users could seek the options which give high quality results, less cost and less time. Additionally to that, each user tries to subtract a lot of examples of buildings he previously executed and the pros and disadvantages in order to insist on imposing his opinion on the other person. This perhaps made the conversation might be nonproductive, i.e. the users in FTF talk more because it is really easier speaking in FTF while the users in the CMC might be more concentricity on the task items because each of them was in front of the computer, and do not have the physical occurrence of someone near them to speak. All of these are possibilities, the analysis of productivity given later assistance to specify which of these scenarios is more acceptable.

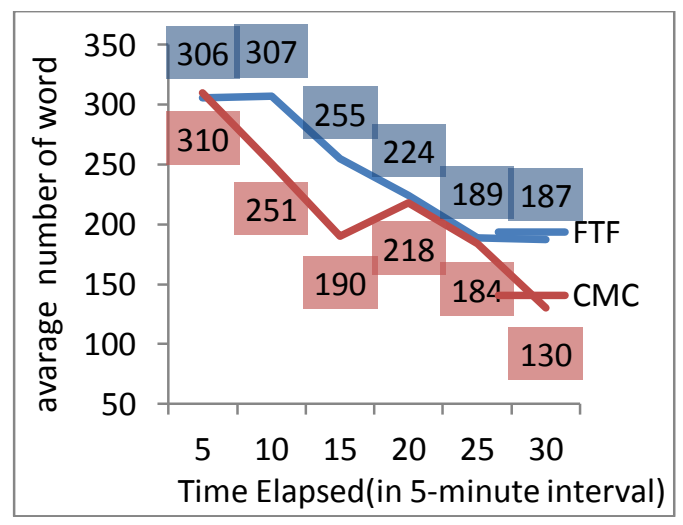

Figure 6: The Total Number of Work Related Words

\subsection{Total Non-work Related Words (TNN- WRWs)}

The words out of work related to social speech that have a significant impact on the team productivity, for example, greeting, the security situation and other speech items. It is clear that in the above two Figure 5 and 6, the (TNN-WRWs) in comparatively is small but it is interesting to note that (TNN-WRWs) distribution with time as shown in Figure 7. Again the results are medium for all the experiments. The (TNN-WRWs) in FTF is 351 and 194 in CMC giving a percentage of the spoken of $13.16 \%$ in $\mathrm{CMC}$ and $19.29 \%$ in FTF. The average of (TNN-WRWs) over the six 5-minute intervals in FTF is 58.5 words every 5-minutes while 32 for CMC. This was stated by the statistical analysis ( Student's tdistribution) where there is the significance statistical difference between the two mediums of the two groups.

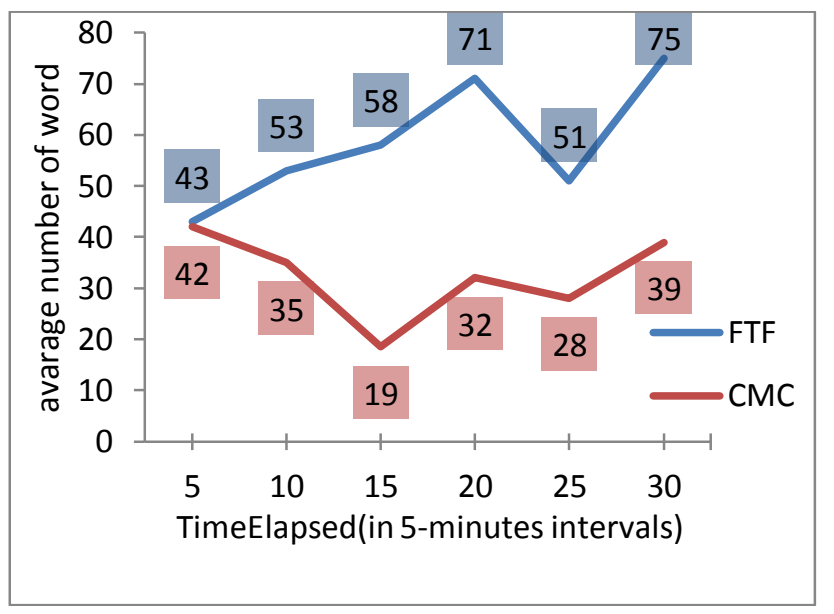

Figure 7: Non-Work Related Words in FTF and $\mathrm{CMC}$

The calculate value of $\mathrm{t}$ (" $\mathrm{t}$ Stat") is 4.29 , This value is compared to the value of "t Critical two-tail" (tcrit) of 2.13 based on $\alpha=0.05$ (one-sided), and $15 \mathrm{df}$. Because("t Stat") is bigger than tcrit (4.29> 2.13). Thus, (TNNWRWs) medium of FTF is measured to greater than non-work Related Words medium of CMC at the $95 \%$ level of confidence.

However the two curves exhibit distinct differences to those of (TNN-WRWs). In FTF, generally this number increases with time as shown in the Figure 7. It is relatively less number than (TNN-WRWs) in the beginning of the experiment, this is due to the users are more serious in their work, but the curve of (TNN-WRWs) in the passage of time (except minute 25) decreased suddenly and then increased again .This means that there is an increase in social interaction, releasing of psychological pressure, especially they are in front the camera, because most of the people 
in Iraq are a void of cameras. In CMC, the trend of the curve decrease with the time until the $15^{\text {th }}$ minute, then it was still fluctuated to the end of the experiment .In the beginning of the experiment, there is relatively more (TNN-WRWs)because the users need to know eachother in long distance conversations. It is obvious that the (TNN-WRWs)were increasing with people that have previous knowledge of each other as compared to those who were strangers.

\section{4 .Working Time}

During the observing of video recording and audio track of the sixteen experiments, it is impossible spending experiment time in working only, because some users try to speak out of the subject, speak with other people and eat . This could be to attributed into behavior of peoples and they may not be able to continue their work without joking. Therefore ,we divided the time into three categories, working time, wasting time and undefined time.

Working Time consists of time spent on a (TNWRWs) and time spent on making changes in the model. As it was before, the results are the averages of 16 experiments. Generally, it is shown in Figure 8, the (WT) of CMC is little higher than that of FTF. This explains that CMC is more productive than FTF or that $\mathrm{CMC}$ requires more effort because the additional load imposed by the limitations of the communications. Over the six 5-minute intervals. The users in FTF spent an average (WT) of 252 seconds every 5-minutes while in CMC they spent 262 second, This is stated by the statistical analysis (the Student's tdistribution) .

The calculation value of $t$ ("t Stat") is 2.93347, this value is compared to the value of "t Critical two-tail" (tcrit) of 2.13 based on $\alpha=$ 0.05 (one-sided), and $15 \mathrm{df}$. Because("t Stat") is greater than tcrit $(2.93>2.13)$. Thus, Working time medium of FTF is measured less that the working time medium of CMC at the $95 \%$ level of confidence.

It is also thrilling to look at the distribution of working time in FTF and CMC in Figure 8, the working time in CMC is more than in FTF, the users were more serious in working because they are working with relax, without tension. Each of them does not have enough time to talk off-topic, as well as they try to focus on completing the task .Specially, In some FTF experiment, the users are different in gender, there would be a problem in their behavior and that would make the female more shy during the experiments and this leads to decrease the working time and productivity while in CMC this factor would be less effective .As shows Figure 4.6, the distribution of work activity with time is not similar between FTF and CMC, As previously mentioned in explanation of Figure 5, users in FTF discuss most of the workset and after agreement on most worksets they start changing on computer, it is clear that the users had a strong start and then their productivity decrease with time to end the experiment. In CMC each workset is discussed a lone and then they begin to change, the curve of working time decrease until the $15^{\text {th }}$ minute then it increases to the $20^{\text {th }}$ minute and then decrease gradually to the minimum value in the end of the experiment , The researcher explains that the working time is high when users discuss between them ,but when they begin to change on the model, working time is less, that goes back to the fact that the users are not familiar with Revit and all that was lead to a time wasting.

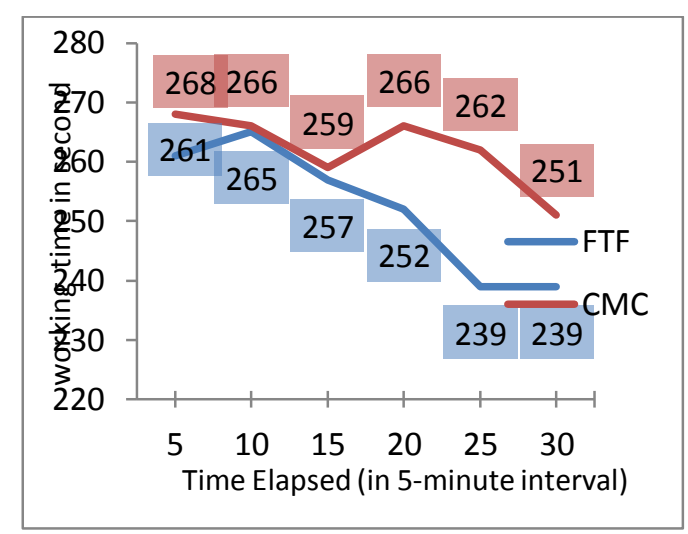

Figure 8: Working Time in FTF and CMC

\subsection{Wasted Time}

Wasted time is defined as the time which is spent on (TNN-WRWs). This time can be extracted from analyzing the video recording directly. As can be seen from the outcomes offered in Figure 9, wasted time for FTF is consistently higher than wasted time in CMC . The averages of wasted time spent for every 5 minutes were 32 second in FTF and 21 in CMC. This is due to that FTF is more easy than CMC in terms of social speak, especially if the users have a previous relationship i.e Familiar with each other's, while in CMC, the situation is totally different comparing with FTF . Also, this is stated that the statistical analysis (Student's t-distribution ) where there are the significant statistical difference between the mediums of the two groups.

The calculation value of $t$ ("t Stat") is 2.87 This value is compared to the value of " $t$ Critical two-tail" (tcrit) of 2.13 based on $\alpha=$ 0.05 (one-sided), and $15 \mathrm{df}$. Because("t Stat") is bigger than tcrit $(2.87>2.13)$. Thus, Wasted time medium of FTF is measured to greater 
than the Wasted time medium of CMC at the $95 \%$ level of confidence.

Wasted time spent by users in FTF differs from $\mathrm{CMC}$; this could be the users in $\mathrm{CMC}$ is slightly better than FTF in utilizing the time to complete the task within specific time as shown in the results above. It has been found useful to discuss the distribution of this time between the two groups of users and what was the link between this time and behavior for each user during FTF and CMC.

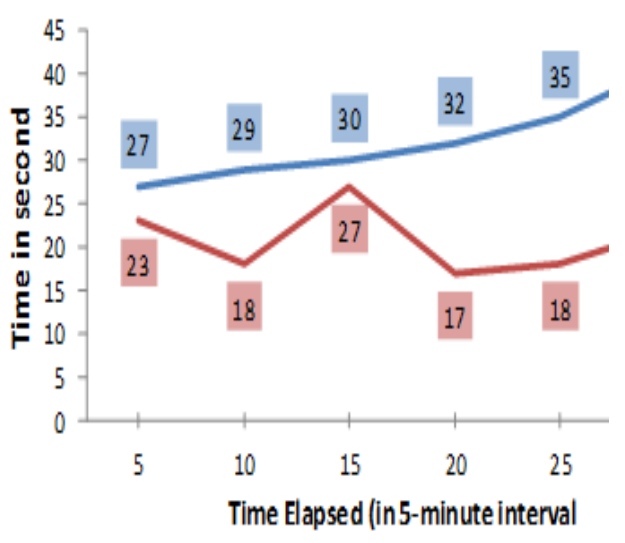

Figure 9: Waste Time in FTFand CMC

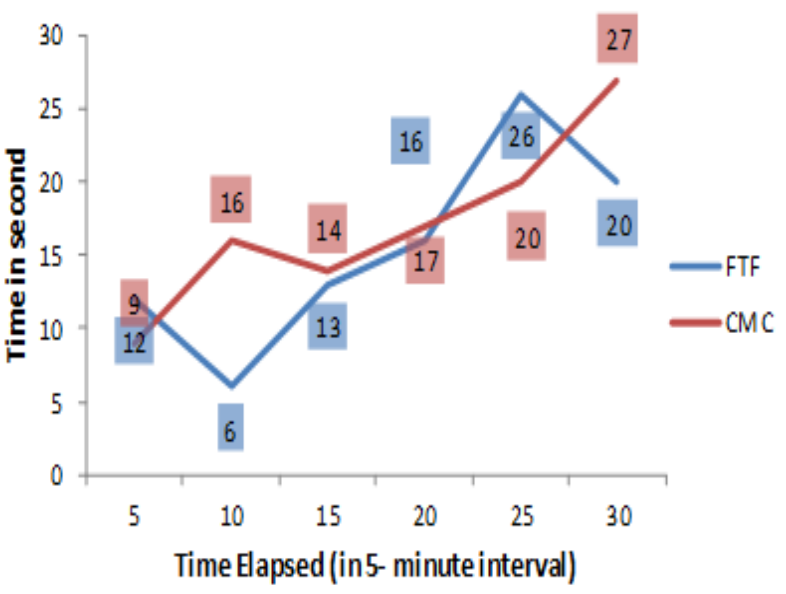

Figure 10: Undefined Time in FTF and CMC

\subsection{Undefined Time}

From the analysis of the video recordings , undefined time is defined as the time that is unknown whether it is a working or wasting time .Therefore, in this section, and some of the other next sections, this classification will be studied to see if it should be connected with working time or wasted time. However, analysis of the class (especially productivity in next section) of the time can state whether it is side working or wasted-time. If this time has taken the same trend of wasted time (i.e. decrease the productivity in every time interval) this means that it is wasted time, But if this time increases the productivity in every time interval, this means that it is working time. As in Figure 10, undefined time is slightly higher in CMC than FTF. To try to obtain a better thought of what is happening in undefined time (i.e. is it work related or not), the link between the general trend of the undefined time and team productivity for every experiment has been investigated for each time interval. It can be concluded that undefined time shows the type of working time in 10 of the 16 FTF experiments, and in the remaining 6 experiments it was a wasting time. For CMC, undefined time shows the type of working time and in 6 of the $16 \mathrm{CMC}$ experiments, and in remaining 10 experiments it is wasting time. Generally undefined time in FTF was 93 seconds per 30 minutes experiment ; while in
CMC it was 103 seconds. The averages of undefined time spent every 5 minutes in FTF was $16 \mathrm{Sec}$ and for $\mathrm{CMC}$ was $17 \mathrm{Sec}$. There is no significant statistical difference between the two averages .

\subsection{The Number of Exchanges(NOE)}

The (NOE) is defined as it is the number of times, in which, utterance exchanges between users through the experiment (i.e. One person breaks talking and another start after). Interaction between users depends on the relationship between them .Figure 11 illustrated that the total (NOE) in CMC is higher than in FTF because more persons are more interacting when using CMC. These results were consistent with the finding of Rice, L. \& Markey [23], who stated that the interaction between the users in FTF was less than in CMC. In all experiments FTF and $\mathrm{CMC}$, interaction between users is increasing with the increasing number of words, also it is obvious in FTF that interaction is being decreased between them in the passage of time that goes back to the decreasing cohesion between them . It is obvious in minute 20, 25,30 that the (NOE) in CMC is higher than in FTF where the users are more focused on the task. The average of the (NOE) between the users for every 5 minutes in FTF is 17 while it is 18 in CMC . There is no significant statistical difference between the two medium because the t-value of the "two-tailed test" from the analysis of the results is -2.125 , this is being less than the $t$-critical value of 2.13 with a degree of freedom of $\mathrm{df}=15$, with a probability of error $>5 \%$. However, absolutely, no one can say that this difference 
is due to a higher level of collaboration for CMC.

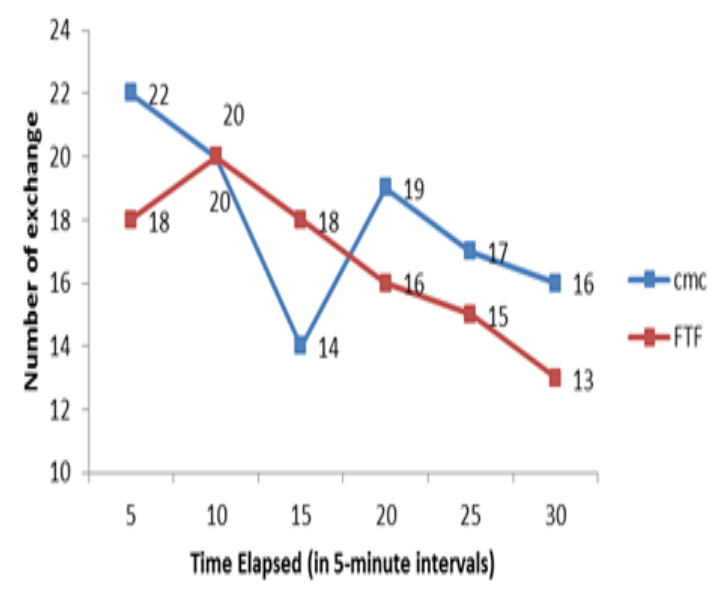

Figure 11: Total Number of Exchanges in FTF and $\mathrm{CMC}$

\subsection{Productivity}

The evaluation of productivity for both teams and every user is by specifying points for several features of the task which have been accomplished successfully. As shown in Figure 12, the team productivity in CMC is higher than in FTF in most time intervals, in the $15^{\text {th }}$ minute FTF was higher as well as the productivity in the first part of the experiment is nearly higher than the second part of the experiment. This indicates that the users made a good productivity but with the time their productivity decreased. For the cumulative productivity, both curves have the same trend in most points and team productivity in CMC is higher than is FTF.

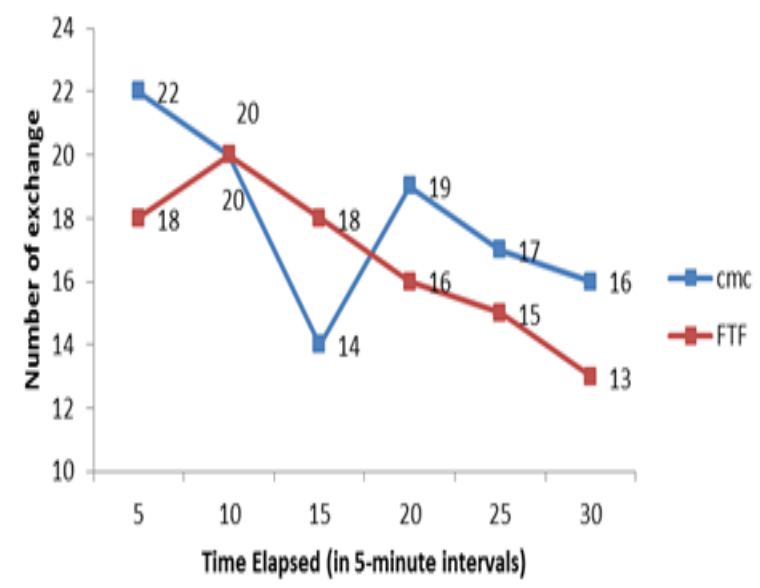

Figure 12: Productivity and Cumulative Productivity in FTF and CMC

\section{Conclusions}

5.1 Quantity of Communication

5.1.1 Total number of words words is a measure of the quantity of communication done. The average of (TNOW)in FTF has recorded 303 words per 5-minutes while in CMC the total number of words has recorded 246 words, the total number of words said by each team in FTF is $23 \%$ higher than in CMC during the six 5-minute intervals.

\subsection{2-Total Number of Work Related Words (TNWRWs)}

The (TNN- WRWs) in FTF was 351 and 194 in CMC, giving a percentage of the total words spoken $13.16 \%$ in CMC and $19.29 \%$ in FTF. The average of (TNN- WRWs)over the six 5minute intervals in FTF was 58.5 words every 5-minutes while 32 for CMC, that is because there were more increasing in social interaction in FTF than in CMC. The results which were concluded from this research are that communication quantity in FTF were more than in CMC, but percentage of (TNWRWs) in $\mathrm{CMC}$ were more than in FTF and percentage of (TNN- WRWs) in CMC were less than in FTF, this indicated that communication in CMC might be more productive than communication in FTF. This might be considered as the most important results in this research .

\subsection{Time Consumed}

5.2.1 -Working time : The users in FTF spent an average working time 252 seconds every 5minutes while in CMC they spent 262 seconds, the working time in CMC was a little more than in FTF. This explains that CMC was slightly more productive than FTF .

5.2.2- Wasted Time : wasted time in FTF regularly more than in $\mathrm{CMC}$. The averages of wasted time for every five minutes was 32 seconds in FTF and 22 in CMC. This is due to that FTF was more easy than CMC in terms of social speech, especially if the users were having a previous relationship i.e Familiar with each other's.

5.2.3- Undefined time : it was distributed between wasting and working time. It can be concluded that undefined time shows the type of working time in 10 of the 16 in FTF experiments, and in the remaining 6 experiments it was a wasted time. For CMC, undefined time shows the type of working time and in 6 of the $16 \mathrm{CMC}$ experiments, and in remaining 10 experiments it is wasted time. The reason was that communication management in CMC requires more effort.

\subsection{Degree of Collaboration}

\subsubsection{The Total Number of Exchanges (TNOE):}

TNOE in CMC was more than in FTF because most of persons were having more interaction when using CMC . The average of the number 
exchanges between the users for every 5 minutes in FTF was 17 while it was 18 in CMC. Absolutely no one can say that this variance was because of a higher level of collaboration in CMC. These results were agreed with the finding of Rice \& Markey (2009).

\subsection{Productivity}

The cumulative productivity in CMC was 8.32 while in FTF it was 7.19, This indicates that CMC communication provides a good environment for collaborative work which increases team productivity, this result is agrees with Hatem .

\section{Reffernces}

[1]. Rajendran, P., Seow, T. W., \& Goh, K. C. Bulding Information Modeling (BIM) in design stage to assist in time, cost and quality in construction innovation, International Journal of Conceptions on Management and Social Sciences, 2, Issue. 3, (2013), ISSN: $2357-2787$.

[2]. Arayici, Y., Egbu, C. O., \& Coates, P. (2012). Building information modelling (BIM) implementation and remote construction projects: issues, challenges, and critiques. Journal of Information Technology in Construction, 17, 75-92.

[3]. Azouz, Z., Katsanis, C. J., Forgues, D., Aksenova, G., Poirier, E., \& Doré, S. The BIM Utopia: Centralizing Collaboration and Communication through Technologies, Working Paper Proceedings Engineering Project Organization Conference Devil's Thumb Ranch, Colorado, 2014.

[4]. Project Management Institute (2008), A guide to the project management body of knowledge (PMBOK® Guide), Newtown Square, Pa., Project Management Institute, Inc. [5]. PMI, THE HIGH COST OF LOW PERFORMANCE:THE ESSENTIAL ROLE OF COMMUNICATIONS, 2013.

[6]. Burke, R, Fundamentals of project management: tools and techniques. Ringwood: Burke Pub, 2010.

[7]. Alshawi, M. and Ingirige, B. Web-enabled project management: an emerging paradigm. Automation in Construction. 12 (4), (2002), 349-64.

[8]. Scanlin, J. The Internet as an enabler of the Bell Atlantic project office, Project Management Institute ,June, 1988.
[9]. Ean L. Cheng ,Face-to-face Versus Computer-mediated Communication: Exploring Employees' Preference of Effective Employee Communication Channel, International Journal For The Advancement Of Science \& Arts, 1 (2), (2010).

[10]. Martins L.L. , Gilson L.L., and Maynard T.M., Virtual teams: What do we know and where do we go from here? Journal of Management, 30(6), (2004), 805-835.

[11]. Leenders, R.T.A.J., J.M.L.V. Engelen and J. Kratzer, Virtuality, communication, and new product team creativity: a social network perspective. Journal of Engineering and Technology Management, 20(2003), 69-92. [12]. Bell B.S., and Kozlowski S.W., A typology of virtual teams: Implications for effective leadership, Group and Organization Management, 27(1),(2002), 14-49.

[13]. Bell, S. T., Villado, A. J., Lukasik, M. A., Belau, L., \& Briggs, A. L. Getting specific about demographic diversity variable and team performance relationships: A meta-analysis. (Electronic Version). Journal of Management , 37 (3), May (2011), 709-743.

[14]. Duarte, D. L., \& Snyder, N. T. Mastering virtual teams: Strategies, tools, and techniques that succeed. John Wiley \& Sons, 2006.

[15]. Kratzer, J., Leenders, R. T. A., \& van Engelen, J. M. Keeping virtual R\&D teams creative, Research-Technology Management, 48(2), (2005), 13-16.

[16]. Baan.A, and M. Maznevski, Training for virtual collaboration: Beyond technology competencies, in J. Nemiro, M. Beyerlein, L. Bradley, and S. Beyerlein (Eds.), The handbook of high performance virtual teams: A toolkit for collaborating across boundaries ,(San Francisco: Jossey Bass, (2008) ,345-365.

[17]. Andres, H. P. A comparison of face-toface and virtual software development teams. Team Performance Management: An International Journal, 8(1/2), (2002), 39-48.

[18]. Ojasalo, J. Management of innovation networks: a case study of different approaches, European Journal of Innovation Management, 11(1), (2008), 51-86.

[19]. Hambley, L. A., O’Neill, T. A., \& Kline, T. J. Virtual team leadership: The effects of leadership style and communication medium on team interaction styles and outcomes. ScienceDirect - Organizational behavior and 
human decision processes, 103(1), (2007), 120.

[20]. Wakefield, R. L., Leidner, D. E., \& Garrison, G. Research note-a model of conflict, leadership, and performance in virtual teams, Information Systems Research, 19(4), (2008), 434-455.

[21]. Warkentin, M. E., Sayeed, L., \& Hightower, R. Virtual teams versus face-toface teams: an exploratory study of a Webbased conference system. Decision Sciences, 28(4), (1997), 975-996.

[22]. Hatem , W. A. Comparing the effectiveness of face to face and computer mediated collaboration in design, (Doctoral dissertation, Cardiff University United Kingdom), 2012.

[23]. Rice, L. \& Markey, P. M., The role of extraversion and neuroticism in influencing anxiety following computer-mediated interactions, Personality and Individual Differences, 46(1), (2009), 35-39. 